\title{
Alginate-Based Hydrogel Beads as a Biocompatible and Efficient Adsorbent for Dye Removal from Aqueous Solutions
}

\author{
Safoura Asadi, Setareh Eris, and Saeid Azizian*(i) \\ Department of Physical Chemistry, Faculty of Chemistry, Bu-Ali Sina University, Hamedan 65167, Iran \\ Supporting Information
}

ABSTRACT: In this study, sodium alginate was employed as a starting material for preparing two kinds of biocompatible adsorbents, including calcium alginate hydrogel beads and magnetic hydrogel beads. Fourier transform infrared spectroscopy, X-ray diffraction pattern, and scanning electron microscopy/energy-dispersive $\mathrm{X}$-ray techniques were used to characterize the prepared adsorbents. The performance of the prepared adsorbents for the removal of methyl violet from aqueous solution was studied in detail. Both kinetics and equilibrium aspects of methyl violet adsorption were investigated, and the obtained equilibrium and kinetics data were described with various adsorption models. The effects of initial dye concentration, adsorbent dosage, and temperature on adsorption performance were investigated. Thermodynamic parameters of adsorption were obtained as well.

\section{INTRODUCTION}

In the last century, the massive growth of population on one hand and the rapid industrialization on the other resulted in release of different pollutants in the environment, including dyes. Dyes are synthetic compounds which are widely used in cosmetics industry, paper, plastic, and so on. ${ }^{1,2}$ Therefore, the wastewater of such industries contains high concentration of dyes. Discharging of this wastewater into the water sources reduces water quality and affects aquatic living organism, damages human health, and aesthetic nature of the water. Therefore, the removal of synthetic dyes from water is environmentally important.

Removal of dye compounds from aqueous solutions has been widely studied, and several physical and chemical techniques have been proposed for purification of water from these compounds including photocatalysis, oxidation, filtration, coagulation-flocculation, ozonation, and adsorption. ${ }^{3-5}$ Among all of these techniques, adsorption is considered as one of the most useful methods for the removal of dyes because its operation is facile and efficient and the adsorbent can be recovered and reused. ${ }^{6,7}$ Dissolved dye molecules can bind to the surface of the adsorbent by physical or chemical bonds. 8

Methyl violet (MV) is one of the poison dye compounds, which is used in textile and paper dyeing and so on (the chemical structure of MV is shown in Figure S1). Various adsorbents have been applied for the removal of MV from wastewater such as membrane ${ }^{9}$ waste materials, ${ }^{10}$ chitosan, ${ }^{11}$ agricultural waste, ${ }^{12}$ sepiolite, ${ }^{13}$ fly ash, ${ }^{14}$ perlite, ${ }^{15}$ and powdered activated carbon. ${ }^{16}$ However, most of these adsorbents are neither cost effective nor biodegradable. ${ }^{17,18}$
To improve these weaknesses, a new class of adsorbents, biopolymer-based adsorbents, are introduced in water purification applications. In recent years, many studies have been carried out on using biopolymers as the adsorbent because of their simple synthesis, environmental compatibility, biodegradability, and low cost.

Also, these materials can be modified for specific pollutant removal. ${ }^{19,20}$ Biopolymers and, in particular, sodium alginate are biodegradable compounds that have many hydroxyl and carboxylate functional group in their internal structure. ${ }^{21-23}$ Therefore, because of the presence of numerous anionic groups, they can be considered as a new candidate for the removal of cationic pollutions from waste water (similar to cationic dyes). Dye adsorption on biopolymer-based adsorbents occurs through electrostatic attractions between adsorbents and contaminants. ${ }^{24}$

Sodium alginate, a natural water-soluble salt of alginic acid, is a linear polysaccharides that is extracted from brown algae. It has high bioavailability, and its extraction process is easy. This natural polymer consists of $\alpha$-L-guluronic (G) and $\beta$-Dmannuronic (M) acid residues (Figure 1), and these produce a variety of sequential 1,4-linkage with other materials. The ability to form hydrogels is one of the main properties of alginate; this property of sodium alginate is mainly because of the substitution of sodium ions from the guluronic acid residues by different divalent cations $\left(\mathrm{Ca}^{2+}, \mathrm{Sr}^{2+}, \mathrm{Ba}^{2+}\right.$, etc.). Therefore, a $3 \mathrm{D}$ network is formed as a result of binding

Received: September 24, 2018

Accepted: October 26, 2018

Published: November 9, 2018 


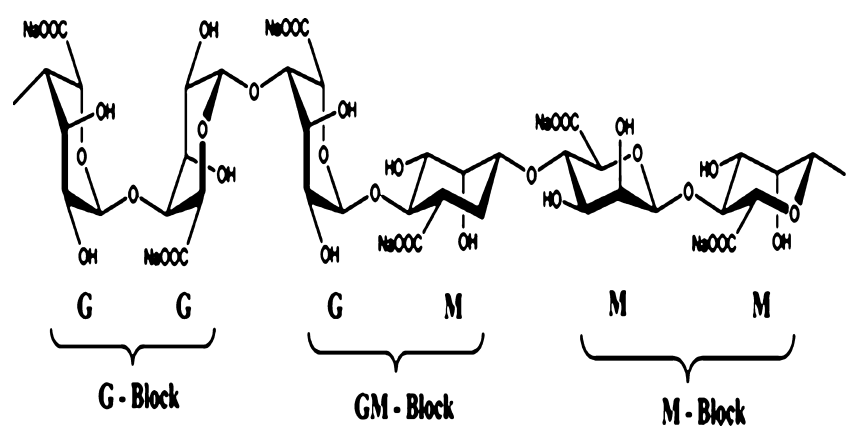

Figure 1. Chemical structure of sodium alginate.

divalent cation to the $\alpha$-L-guluronic block (and between two different chains) (Figure 1). ${ }^{25,26}$ Hydrogels of calcium alginate can be used as a potential sorbent because of its low cost, hydrophilicity, nontoxicity, and biocompatibility.

Introducing nanoparticles into the biopolymer matrices can significantly improve thermal, chemical, mechanical, and surface properties of biopolymers. ${ }^{27,28}$ Recently, interests in biopolymer-based metal oxide nanoparticles have been increased because of biocompatibility, their ability for the removal of dyes and other containments from water, and so on.

Magnetic nanoparticle (MNP) adsorbents based on iron oxide have received great attention. This attention is because of the fundamental properties of MNPs such as particle size and large surface area. ${ }^{29}$ One of the most important advantages of using magnetic-based adsorbent is the ease of separation from treated water through an external magnetic field. This property leads to a possibility of reusing the adsorbent (and also recovering the adsorbed substrate). ${ }^{30,31}$ Preparation of nanocomposites of calcium alginate polysaccharides with iron oxide provides materials which have desired properties of good adsorbents. $^{26,32}$

In this study, calcium alginate and magnetic composite beads were prepared and used as adsorbents for the removal of MV from aqueous solution. Adsorption of MV was studied from equilibrium and kinetics viewpoints. Furthermore, the effect of different parameters including temperature, adsorbent mass, and initial concentration of MV was studied too.

\section{RESULTS AND DISCUSSION}

2.1. Samples Characterization. Scanning electron microscopy (SEM) images of the synthesized iron oxide are shown in Figure 2.

Figure 2a,b shows that the as-synthesized iron oxide structures are microspheres with a diameter of $\approx 1.5 \mu \mathrm{m}$. Figure $2 c$ shows that each microsphere is composed of several nanosheets. Figure $2 \mathrm{~d}$ shows that the nanosheets are formed by aggregation of nanoparticles $(15-20 \mathrm{~nm})$. Figure 3 represents the SEM images of dried magnetic beads. This figure clearly shows that the iron oxide microparticles were coated with a thin layer of calcium alginate.

From the results of the EDX (energy-dispersive X-ray) spectra of iron oxide (Figure S2a), it can be found that the synthesized iron oxide is only composed of iron $(\mathrm{Fe})$ and oxygen $(\mathrm{O})$. Furthermore, the EDX spectrum of the dried magnetic beads is shown in Figure S2b, which confirms the presence of oxygen $(\mathrm{O})$ and iron $(\mathrm{Fe})$ elements. Also, the appearance of $\mathrm{C}$ and $\mathrm{Ca}$ peaks confirms the presence of calcium alginate layer.
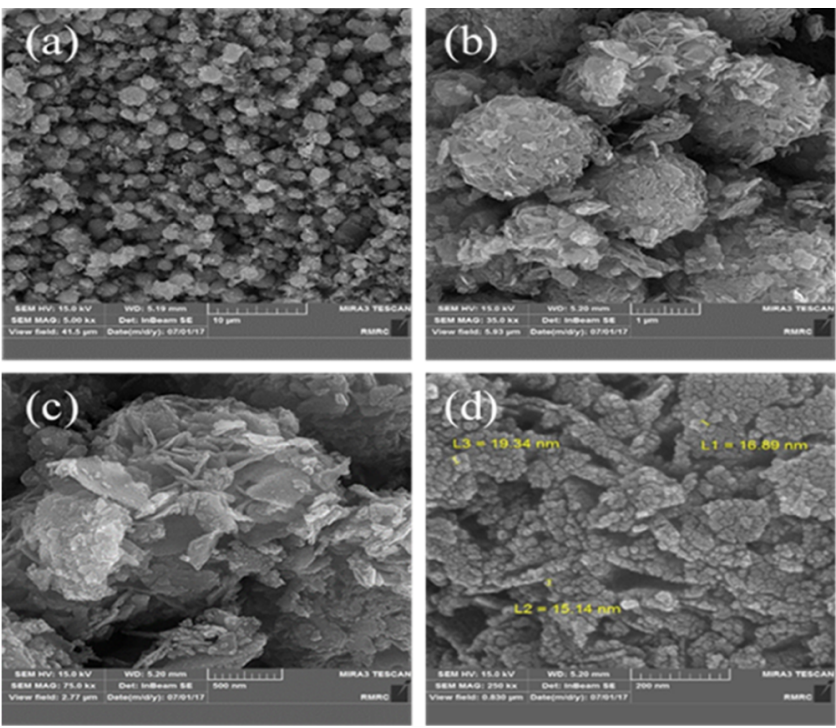

Figure 2. SEM images of the synthesized iron oxide with increasing magnification from $(a-d)$.
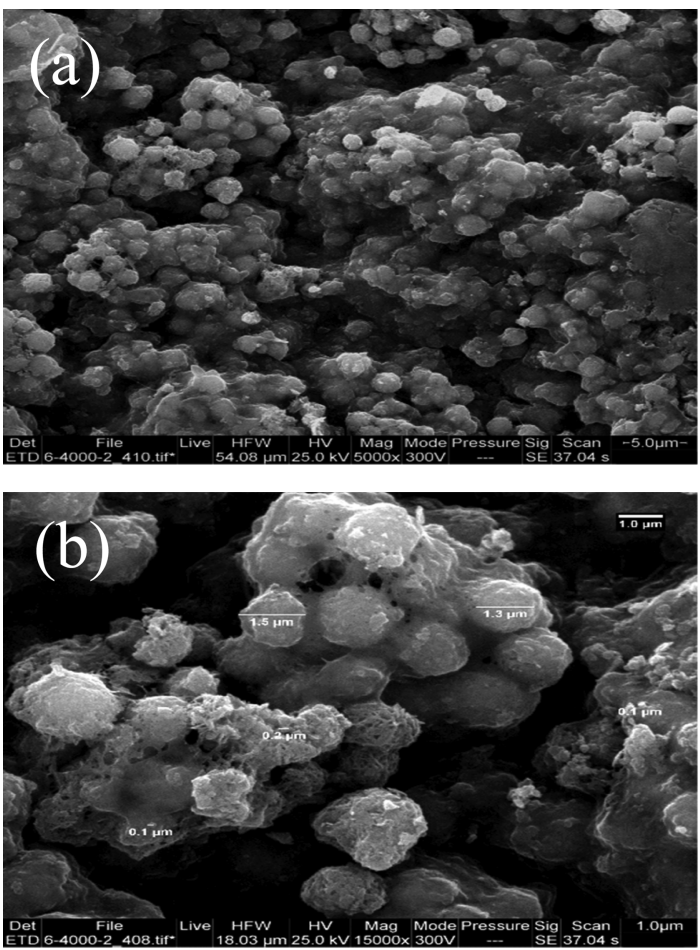

Figure 3. SEM images of dried magnetic beads with (a) low and (b) high magnifications.

Figure 4 shows the X-ray diffraction (XRD) pattern of the dried magnetic composite hydrogel beads. The XRD pattern shows six characteristic peaks of $\mathrm{Fe}_{3} \mathrm{O}_{4}$ at $2 \theta=30.1,35.8,43.1$, $53.9,57.2$, and $63.1^{\circ}$ that can be indexed to (220), (311), (400), (422), (511), and (440) planes of $\mathrm{Fe}_{3} \mathrm{O}_{4}$, and a characteristic peak at $2 \theta=33.0^{\circ}$ that can be assigned to (104) plane of $\alpha-\mathrm{Fe}_{2} \mathrm{O}_{3}$. Therefore, from the XRD pattern, it can be concluded that the synthesized iron oxide is composed of $\mathrm{Fe}_{3} \mathrm{O}_{4}$ and $\alpha-\mathrm{Fe}_{2} \mathrm{O}_{3} .{ }^{33,34}$

The FTIR (Fourier transform infrared) spectrums of calcium alginate beads, magnetic calcium alginate beads, MV- 


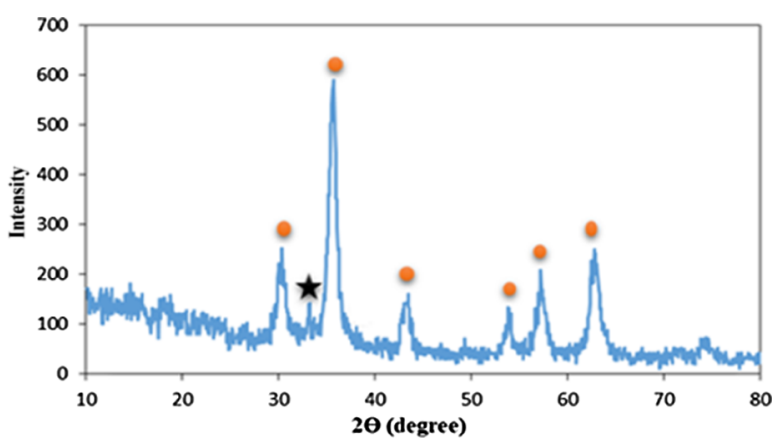

Figure 4. XRD pattern of dried magnetic beads.

loaded calcium alginate beads, and MV-loaded magnetic calcium alginate beads are shown in Figure 5.
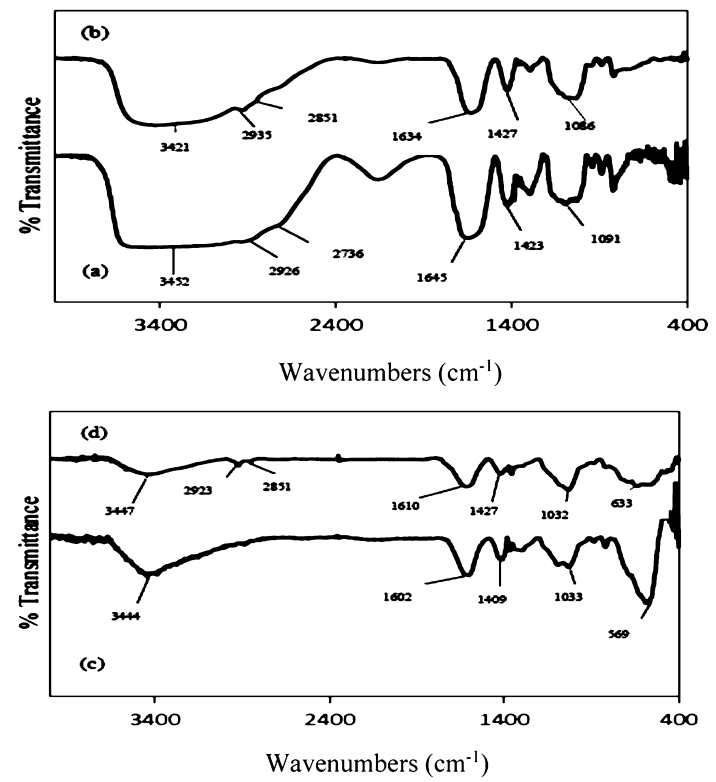

Figure 5. FTIR spectra of: (a) calcium alginate hydrogel beads, (b) MV-loaded calcium alginate hydrogel beads, (c) magnetic hydrogel beads, and (d) MV-loaded magnetic hydrogel beads.

In the FTIR spectrum of calcium alginate beads (Figure 5a), the broad peak at $3452 \mathrm{~cm}^{-1}$ is corresponded to $-\mathrm{OH}$ stretching vibration of calcium alginate, and the peaks at 2926 and $2736 \mathrm{~cm}^{-1}$ belong to symmetric and asymmetric $\mathrm{C}-\mathrm{H}$ stretching, respectively. The bands which appeared at 1645 and $1423 \mathrm{~cm}^{-1}$ are assigned to asymmetric and symmetric stretching vibrations of carboxyl groups of calcium alginate, respectively. The band at $1091 \mathrm{~cm}^{-1}$ attributes to $\mathrm{C}-\mathrm{O}-\mathrm{C}$ group. Figure 5c, which is FTIR spectra of magnetic beads, shows that the band at $3444 \mathrm{~cm}^{-1}$ is because of the $-\mathrm{OH}$ stretching and bending vibration and bands at 1602 and 1409 $\mathrm{cm}^{-1}$ are because of asymmetrical and symmetrical stretching vibration of carboxyl group for magnetic beads. Also, the weak band of bending vibration of $\mathrm{C}-\mathrm{O}-\mathrm{C}$ group is appeared in $1033.07 \mathrm{~cm}^{-1}$

The absorption band at the low-frequency zone of 500-700 $\mathrm{cm}^{-1}\left(569 \mathrm{~cm}^{-1}\right)$ corresponds to the stretching vibration of $\mathrm{Fe}-\mathrm{O}$ bands in iron oxide and the FTIR spectrum of calcium alginate beads and magnetic beads after being loaded with MV. Figure $5 b, c$ shows a shift of the peaks and reduction of peak intensity (especially significant reduction in $-\mathrm{OH}$ and $\mathrm{C}=\mathrm{O}$ bands). These changes imply that MV is chemically adsorbed on the surface of the adsorbents.

2.2. Adsorption Kinetics. The study of adsorption kinetics provides indispensable information about the mechanism and rate of adsorption. Also, kinetic studies are of prime importance to design and operate adsorption equipment. Figure 6 shows the changes in adsorbed amounts with time for
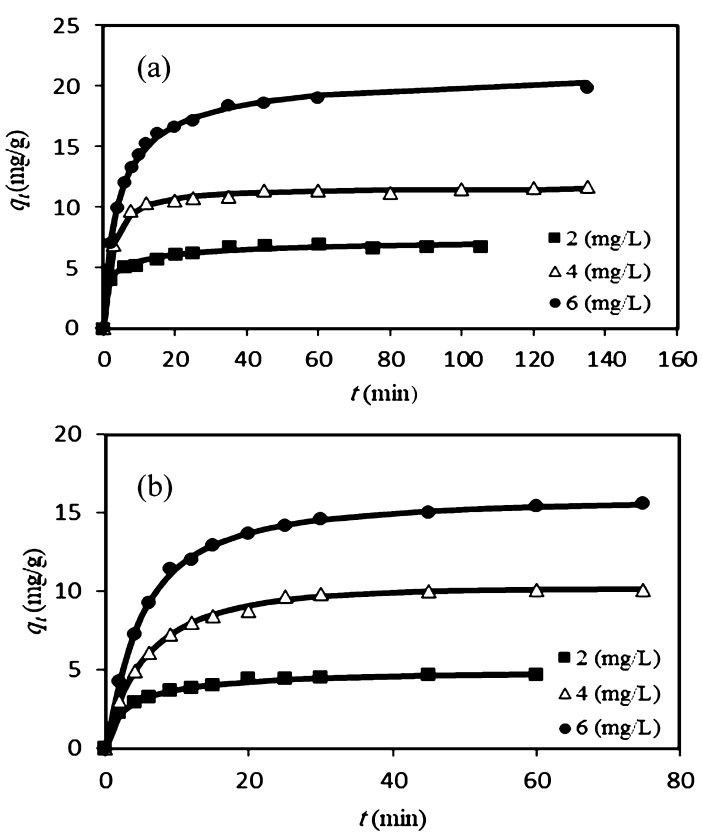

Figure 6. Experimental kinetics data for the adsorption of MV by (a) calcium alginate hydrogel beads and (b) magnetic hydrogel beads at different initial concentrations of MV. The solid lines show the predicted values by the best fitted model.

both types of adsorbents. This figure clearly shows that the adsorption of MV by both types of adsorbents is initially fast, and most of the adsorption occurs within $10 \mathrm{~min}$. Also, it can be seen that by increasing the initial MV concentration, the adsorption rate and capacity increased. Furthermore, from Figure 6, it can be concluded that the rate of MV adsorption on to calcium alginate hydrogel beads is faster than that of magnetic hydrogel beads. The adsorption kinetics data were evaluated using several adsorption kinetics models including pseudo-first order (PFO), ${ }^{35}$ pseudo-second order (PSO), ${ }^{35,36}$ mixed 1,2-order (MOE), ${ }^{37}$ fractal-like pseudo-first order (FLPFO), ${ }^{38,39}$ fractal-like pseudo-second order (FL-PSO), ${ }^{38,39}$ fractal-like mixed 1,2-order (FL-MOE), ${ }^{38,39}$ and Elovich. ${ }^{40}$ The obtained results of data fitting are listed in Tables 1 and S1. The correlation coefficients show that the adsorption kinetic data for MV onto calcium alginate hydrogel beads were better correlated by FL-PSO model. Also, for the adsorption of MV by magnetic hydrogel beads, best kinetic model is FLMOE.

Therefore, it can be said that the kinetics of MV adsorption on both of the adsorption was found to follow fractal-like kinetic models. The fractal-like model implies that the adsorption rate coefficient is time-dependent for these systems. This means that the adsorption pathway (or site) changes with time. $^{38,39}$ The solid lines in Figure 6 show the predicated values by FL-PSO (for calcium alginate hydrogel beads) and FL-MOE (for magnetic hydrogel beads). 
Table 1. Obtained Contacts of Different Kinetic Models for Adsorption of MV on to Calcium Alginate Hydrogel Beads

\begin{tabular}{|c|c|c|c|c|c|c|c|c|c|c|}
\hline kinetic model & equation & $\begin{array}{c}q_{\mathrm{e}} \\
(\mathrm{mg} / \mathrm{g})\end{array}$ & $k$ & $\begin{array}{c}k_{1} \\
(1 / \mathrm{min})\end{array}$ & $\begin{array}{c}k_{2} \\
{[\mathrm{~g} /(\mathrm{mg} \min )]}\end{array}$ & $f_{2}$ & $a$ & $b$ & $\alpha$ & $r^{2}$ \\
\hline \multicolumn{11}{|c|}{$C_{0}=2(\mathrm{mg} / \mathrm{L})$} \\
\hline $\mathrm{PFO}^{35}$ & $q_{t}=q_{\mathrm{e}}\left(1-\exp \left(-k_{1} t\right)\right)$ & 6.44 & & 0.358 & & & & & & 0.9226 \\
\hline PSO $^{35,36}$ & $q_{t}=k_{2} q_{\mathrm{e}}^{2} t /\left(1+k_{2} q_{\mathrm{e}} t\right)$ & 6.85 & & & 0.081 & & & & & 0.9775 \\
\hline $\mathrm{MOE}^{37}$ & $\begin{array}{l}q_{t}=q_{\mathrm{e}}\left(\left(1-\exp \left(-k_{1} t\right)\right) /\left(1-f_{2}\right.\right. \\
\left.\left.\quad \exp \left(-k_{1} t\right)\right)\right)\end{array}$ & 6.67 & & 0.052 & & 0.9 & & & & 0.9733 \\
\hline Elovich $^{40}$ & $q_{t}=(1 / b) \ln (1+a b t)$ & & & & & & 1.390 & 143.34 & & 0.9831 \\
\hline FL-PSO ${ }^{38,39}$ & $q_{t}=k q_{\mathrm{e}}^{2} t^{\alpha} /\left(1+k q_{\mathrm{e}} t^{\alpha}\right)$ & 7.07 & 0.096 & & & & & & 0.550 & 0.9898 \\
\hline FL-PFO ${ }^{38,39}$ & $q_{t}=q_{\mathrm{e}}\left(1-\mathrm{e}^{-k t^{t}}\right)$ & 7.08 & 0.633 & & & & & & 0.408 & 0.9909 \\
\hline FL-MOE $^{38,39}$ & $q_{t}=q_{\mathrm{e}}\left(1-\frac{\exp \left(-k_{1}^{\prime} t^{\alpha}\right)}{1-f_{2} \exp \left(-k_{1}^{\prime} t^{\alpha}\right)}\right)$ & 7.08 & 0.632 & & & 0.001 & & & 0.385 & 0.9913 \\
\hline \multicolumn{11}{|c|}{$C_{0}=4(\mathrm{mg} / \mathrm{L})$} \\
\hline $\mathrm{PFO}^{35}$ & $q_{t}=q_{\mathrm{e}}\left(1-\exp \left(-k_{1} t\right)\right)$ & 11.17 & & 0.297 & & & & & & 0.9850 \\
\hline $\mathrm{PSO}^{35,36}$ & $q_{t}=k_{2} q_{\mathrm{e}}^{2} t /\left(1+k_{2} q_{\mathrm{e}} t\right)$ & 11.73 & & & 0.044 & & & & & 0.9962 \\
\hline $\mathrm{MOE}^{37}$ & $\begin{array}{l}q_{t}=q_{\mathrm{e}}\left(\left(1-\exp \left(-k_{1} t\right)\right) /\left(1-f_{2}\right.\right. \\
\left.\left.\quad \exp \left(-k_{1} t\right)\right)\right)\end{array}$ & 11.44 & & 0.05 & & 0.9 & & & & 0.9953 \\
\hline Elovich $^{40}$ & $q_{t}=(1 / b) \ln (1+a b t)$ & & & & & & 0.989 & 1170.73 & & 0.9725 \\
\hline FL-PSO ${ }^{38,39}$ & $q_{t}=k q_{\mathrm{e}}^{2} t^{\alpha} /\left(1+k q_{\mathrm{e}} t^{\alpha}\right)$ & 11.61 & 0.047 & & & & & & 1 & 0.9962 \\
\hline FL-PFO ${ }^{38,39}$ & $q_{t}=q_{\mathrm{e}}\left(1-\mathrm{e}^{-k t^{a}}\right)$ & 11.45 & 0.538 & & & & & & 0.557 & 0.9940 \\
\hline FL-MOE $^{38,39}$ & $q_{t}=q_{\mathrm{e}}\left(1-\frac{\exp \left(-k_{1}^{\prime} t^{\alpha}\right)}{1-f_{2} \exp \left(-k_{1}^{\prime} t^{\alpha}\right)}\right)$ & 11.24 & 0.047 & & & 0.9 & & & 1 & 0.9953 \\
\hline \multicolumn{11}{|l|}{$C_{0}=6(\mathrm{mg} / \mathrm{L})$} \\
\hline $\mathrm{PFO}^{35}$ & $q_{t}=q_{\mathrm{e}}\left(1-\exp \left(-k_{1} t\right)\right)$ & 19.02 & & 0.153 & & & & & & 0.9555 \\
\hline $\mathrm{PSO}^{35,36}$ & $q_{t}=k_{2} q_{\mathrm{e}}^{2} t /\left(1+k_{2} q_{\mathrm{e}} t\right)$ & 20.70 & & & 0.011 & & & & & 0.9964 \\
\hline $\mathrm{MOE}^{37}$ & $\begin{array}{l}q_{t}=q_{\mathrm{e}}\left(\left(1-\exp \left(-k_{1} t\right)\right) /\left(1-f_{2}\right.\right. \\
\left.\left.\quad \exp \left(-k_{1} t\right)\right)\right)\end{array}$ & 20.09 & & 0.022 & & 0.9 & & & & 0.9936 \\
\hline Elovich $^{40}$ & $q_{t}=(1 / b) \ln (1+a b t)$ & & & & & & 0.366 & 41.30 & & 0.9600 \\
\hline FL-PSO 38,39 & $q_{t}=k q_{\mathrm{e}}^{2} t^{\alpha} /\left(1+k q_{\mathrm{e}} t^{\alpha}\right)$ & 21.31 & 0.013 & & & & & & 0.867 & 0.9983 \\
\hline FL-PFO ${ }^{38,39}$ & $q_{t}=q_{\mathrm{e}}\left(1-\mathrm{e}^{-k t^{a}}\right)$ & 20.42 & 0.317 & & & & & & 0.562 & 0.9947 \\
\hline FL-MOE ${ }^{38,39}$ & $q_{t}=q_{\mathrm{e}}\left(1-\frac{\exp \left(-k_{1}^{\prime} t^{\alpha}\right)}{1-f_{2} \exp \left(-k_{1}^{\prime} t^{\alpha}\right)}\right.$ & 20.75 & 0.029 & & & 0.5 & & & 0.828 & 0.9978 \\
\hline
\end{tabular}

2.3. Effect of Adsorbent Dosage. The effect of adsorbent dosage on adsorption of MV (with the concentration of 6 ppm) was studied using different amounts of adsorbents (0.0013-0.0026 g dry mass of calcium alginate hydrogel beads and $0.0019-0.0039 \mathrm{~g}$ dry mass of magnetic hydrogel beads).

Figure S3 shows the plots of adspecies amount versus time for different adsorbent dosages. As can be seen in these figures, increasing the adsorbent dosage leads to decreasing the adsorbed amount per unit mass of adsorbent. However, as the adsorbent dosage increases, the required time for reaching equilibrium decreases because of increasing the number of active sites on the surface of the adsorbent.

More recently, a simple graphical method was proposed to elucidate the effect of adsorbent dosages on the rate of adsorption process. ${ }^{41}$ The base of this method is the plot of normalized adsorbed amount, $\frac{q_{\mathrm{e}}-q}{q_{\mathrm{e}}}$, versus the normalized time scale, $t[m]^{n}$ (where $m$ is the mass of the adsorbent and $n$ is the order in the adsorbent mass). In fact, the normalization of time was performed by multiplying each point time by the used adsorbed mass (in each experiment), which was raised by an arbitrary power. The value of this power should be changed until the curves overlay. Here, we used this method for finding the order of the adsorbent mass in the rate equation. For this purpose, the plot of $\frac{q_{\mathrm{e}}-q}{q_{\mathrm{e}}}$ versus $t[m]^{n}$ was drawn for the adsorption of $\mathrm{MV}$ on calcium alginate hydrogel beads (or magnetic hydrogel beads). By choosing the correct value for $n$, all curves (for different masses of adsorbent) coincided, as shown in Figure 7.

Therefore, it can be found that the order of mass of calcium alginate beads in the rate equation is around 0.8 and of magnetic hydrogel beads is about 0.5 (Figure 7a,b).

Also, by finding the order of the adsorption with respect to the adsorbent dosage, it is possible to determine the adsorbent rate constant, which is independent of the adsorbent mass. For this purpose, the linear form of extended pseudo-second-order rate model was used ${ }^{41}$

$$
\frac{t[m]^{n}}{q}=\frac{1}{k_{2} q_{\mathrm{e}}}+\frac{t[m]^{n}}{q_{\mathrm{e}}}
$$

where the values of mass independent rate constant, $k_{2}$, and $q_{\mathrm{e}}$ can be obtained from the intercept and the slope of the plot of $\frac{t[m]^{n}}{q}$ versus $t[m]^{n}$, respectively. Figure 8 shows the linear plot of $\frac{t[m]^{n}}{q}$ versus $t[m]^{n}$ for the adsorption of MV onto different dosages of adsorbents. Also, the adsorption kinetic data of MV onto calcium alginate hydrogel beads and magnetic hydrogel beads were correlated with the linear form of classical pseudosecond-order rate model $\left(\frac{t}{q}=\frac{1}{k_{2} q_{\mathrm{e}}{ }^{2}}+\frac{t}{q_{\mathrm{e}}}\right)^{35,36}$ for comparison. ACS Omega 2018, 3, 15140-15148 

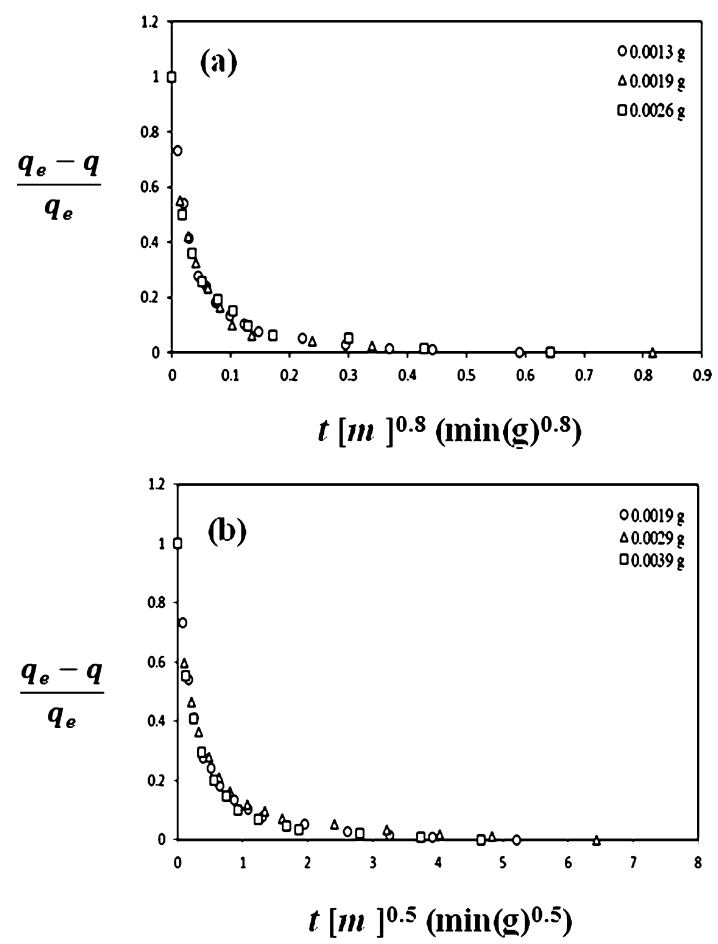

Figure 7. Coincidence of curves for $n$-values of (a) $n=0.8$ for calcium alginate hydrogel beads and (b) $n=0.5$ for magnetic hydrogel beads.
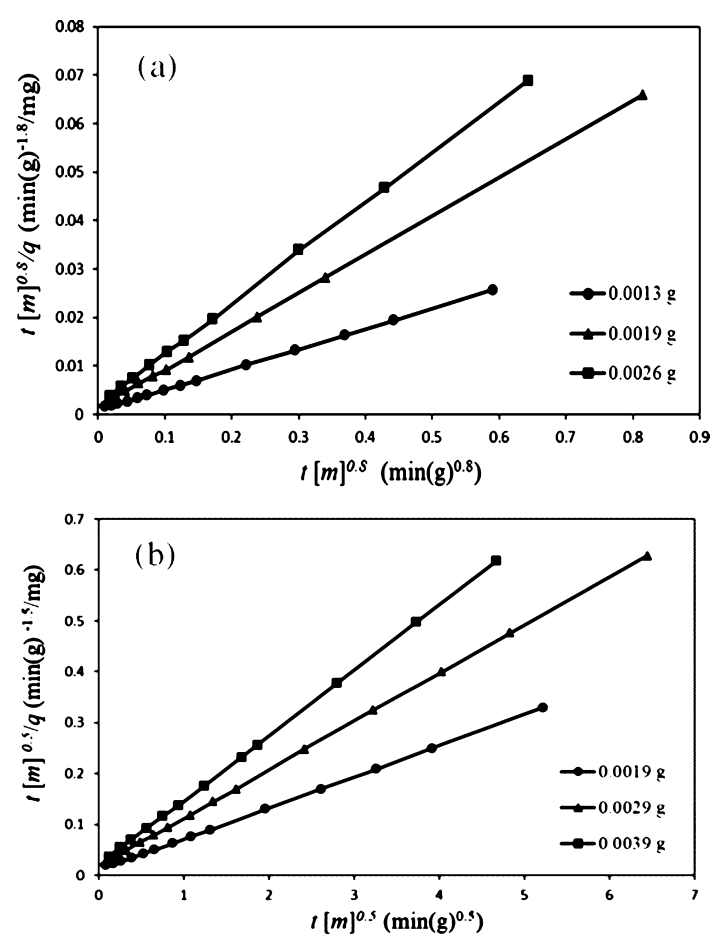

Figure 8. Linear plot of extended pseudo-second-order for the adsorption of MV onto (a) different dosages of calcium alginate hydrogel beads and (b) different dosages of magnetic hydrogel beads.

The obtained values of $k_{2}$ and $q_{\mathrm{e}}$ for these two models are summarized in Table S2.

The obtained results show that in the case of extended pseudo-second-order rate model, the $k_{2}$ does not change with the adsorbent dosage, whereas for classical pseudo-secondorder rate model, it is strongly dependent on the adsorbent dosage. To provide a better visual understanding, the obtained values of $k_{2}$ at different adsorbent dosages by pseudo-secondorder and extended pseudo-second-order models are shown in Figure 9. These figures clearly show that the extended pseudosecond-order model provides the same rate constant for each mass of the adsorbent.
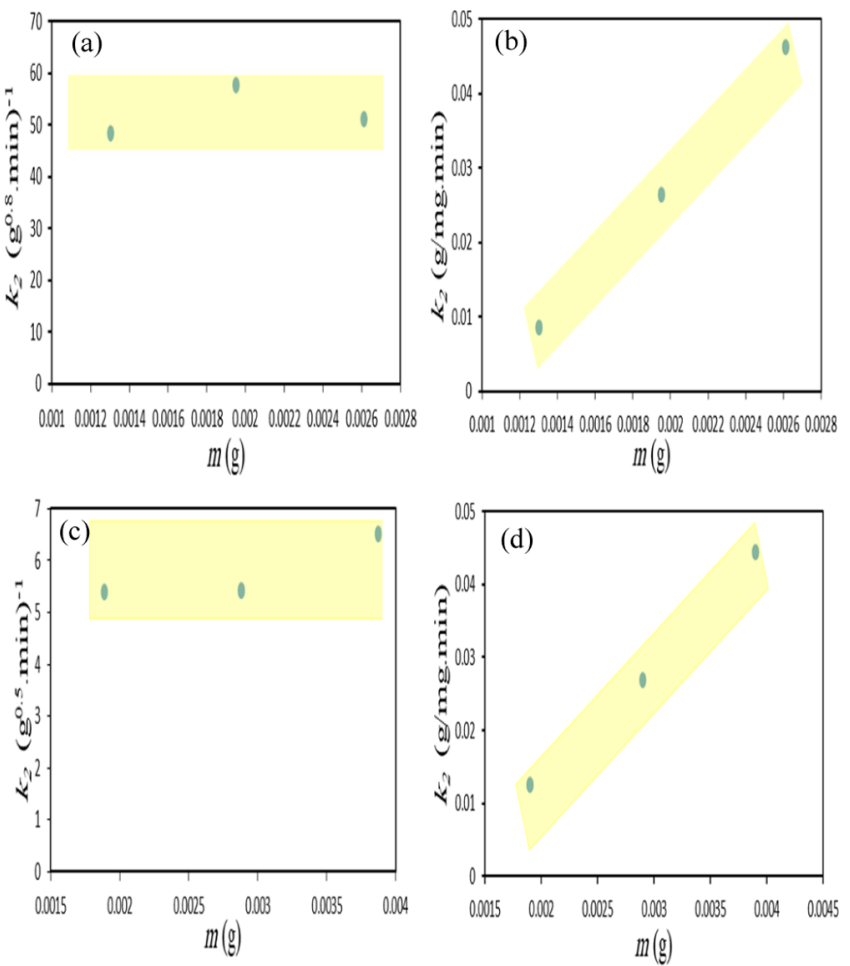

Figure 9. Adsorption rate constants derived from $(a, c)$ extended pseudo-second order and $(b, d)$ pseudo-second order for adsorption of MV on $(\mathrm{a}, \mathrm{b})$ calcium alginate hydrogel beads and $(\mathrm{c}, \mathrm{d})$ magnetic hydrogel beads.

2.4. Adsorption Equilibrium Studies. The knowledge of adsorption isotherms furnishes basic information on adsorbate-adsorbent interactions. The adsorption isotherms are mathematical relationships which describe the distribution of the adsorbate species between the liquid phase and the adsorbed phase at a specific temperature and also at equilibrium conditions.

The equilibrium adsorption data of MV on calcium alginate hydrogel beads and magnetic hydrogel beads are shown in Figure 10a,b. The equilibrium data were modeled with recently presented models including modified Langmuir $(\mathrm{ML})^{42}$ and modified Langmuir-Freundlich $(\mathrm{ML}-\mathrm{F})^{42}$ isotherms and also the classical ones including Freundlich (F), ${ }^{43}$ RedlichPeterson $(\mathrm{R}-\mathrm{P}){ }^{43}$ and Toth $^{43}$ isotherms.

The obtained isotherm parameters along with their correlation coefficients are tabulated in Tables 2 and S3. According to the results, the best correlation coefficients were obtained for ML-F model, indicating that the surface of both adsorbents is heterogeneous. The solid lines in Figure 10a,b show results of fitting by $M L-F$ isotherm. The maximum adsorption capacities of MV, calculated by the ML-F model, are 889 and $713(\mathrm{mg} / \mathrm{g})$ for calcium alginate hydrogel beads and magnetic hydrogel beads, respectively.

2.5. Thermodynamics of Adsorption. Isothermal studies of MV adsorption onto calcium alginate hydrogel were carried 

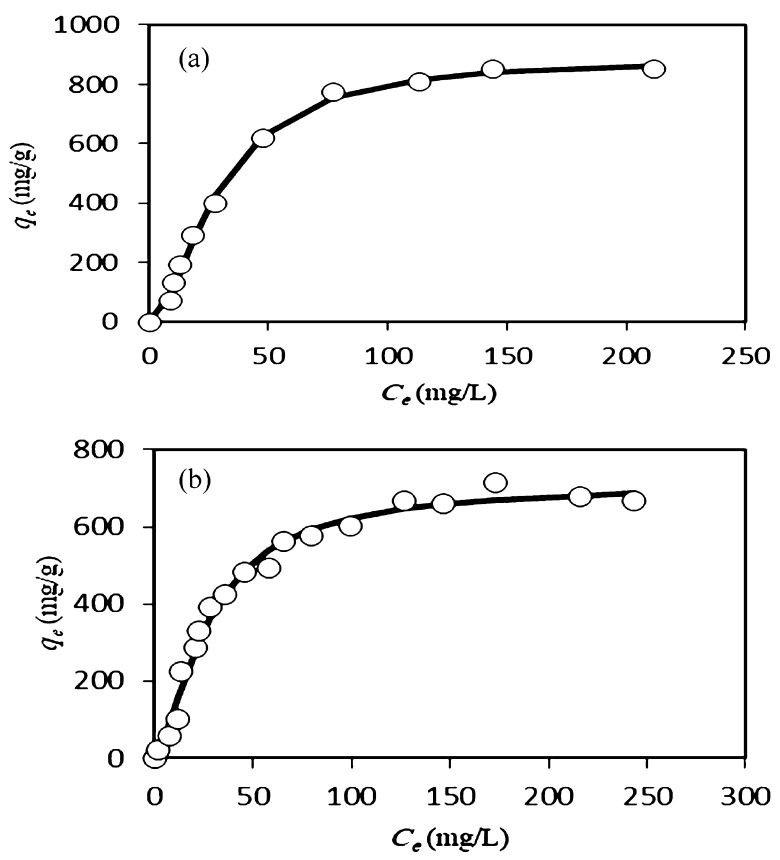

Figure 10. Adsorption isotherm of MV by (a) calcium alginate hydrogel beads and (b) magnetic hydrogel beads. The experimental data are shown with symbols, whereas the solid lines are the predicted values the by $\mathrm{ML}-\mathrm{F}$ isotherm.

out at different temperatures ranging from 25 to $45^{\circ} \mathrm{C}$, and the results are shown in Figure S4. This figure shows that the adsorption capacity of MV onto calcium alginate hydrogel beads decreases slightly with the increasing temperature. The equilibrium data of MV adsorption on calcium alginate hydrogel beads were analyzed with $\mathrm{ML}-\mathrm{F}$ isotherm. The obtained results of this fitting are listed in Table 3.

Also, the thermodynamic parameters [the standard Gibbs free-energy changes $\left(\Delta G^{\circ}\right)$, the standard enthalpy change $\left(\Delta H^{\circ}\right)$, and the standard entropy change $\left.\left(\Delta S^{\circ}\right)\right]$ for the adsorption of $\mathrm{MV}$ adsorption on calcium alginate hydrogel were calculated. The following equation can be applied for the calculation of standard Gibbs free-energy changes $\left(\Delta G^{\circ}\right)^{42}$

$$
\Delta G^{\circ}=-R T \ln K_{\mathrm{MLF}}
$$

where $K_{\mathrm{MLF}}$ is $\mathrm{ML}-\mathrm{F}$ constant (which is a dimensionless parameter). For calculation of the standard enthalpy change $\left(\Delta H^{\circ}\right)$ and the standard entropy change $\left(\Delta S^{\circ}\right)$, the van't Hoff equation was used

$$
\ln K_{\mathrm{MLF}}=\frac{\Delta S^{\circ}}{R}-\frac{\Delta H^{\circ}}{R T}
$$

On the basis of this equation, the values of $\left(\Delta S^{\circ}\right)$ and $\left(\Delta H^{\circ}\right)$ can be derived from the intercept and the slope of $\ln$ $K_{\mathrm{MLF}}$ versus $1 / T$ plot (Figure S5). The obtained values of $\Delta G^{\circ}, \Delta H^{\circ}$, and $\Delta S^{\circ}$ are summarized in Table S4. The obtained results show that the adsorption of $\mathrm{MV}$ on calcium alginate hydrogel beads is endothermic in nature and entropy driven.

\section{CONCLUSIONS}

In summary, the removal of MV by calcium alginate hydrogel beads and magnetic hydrogel beads as adsorbent were studied using batch experiments. It was found that the initial dye concentration, adsorbent dosage, and temperature can

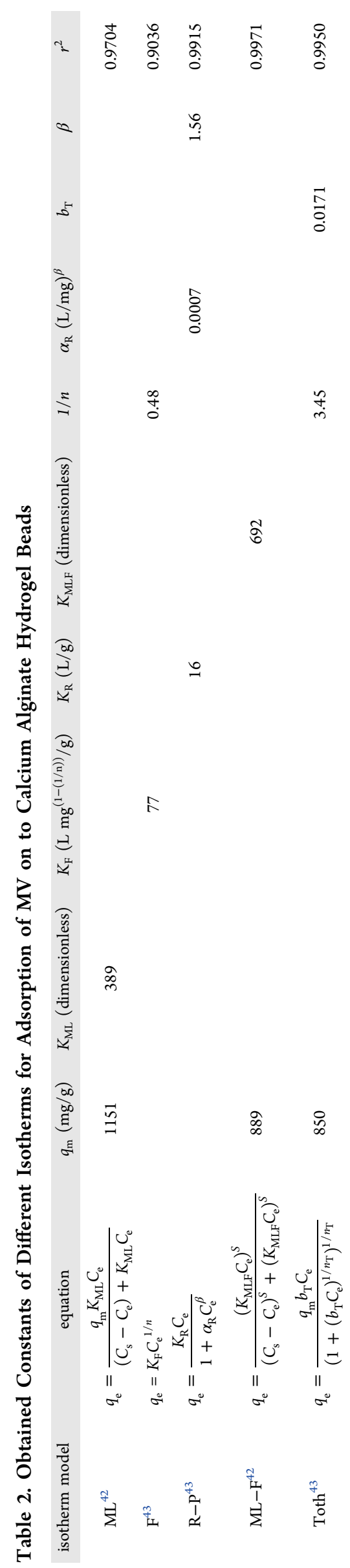


Table 3. Obtained Constants of the ML-F Isotherm for the Adsorption of MV by Calcium Alginate Hydrogel Beads at Different Temperatures

$\begin{array}{cclcc}\text { temperature }(\mathrm{K}) & C_{\mathrm{s}}(\mathrm{g} / \mathrm{L})^{a} & K_{\mathrm{MLF}} & q_{\mathrm{m}}(\mathrm{mg} / \mathrm{g}) & r^{2} \\ 298 & 20.1 & 692 & 889 & 0.9971 \\ 313 & 35.1 & 892 & 886 & 0.9972 \\ 328 & 64.3 & 1447 & 855 & 0.9975\end{array}$

${ }^{a_{T}}$ The values of saturation concentration of MV at different temperatures were obtained experimentally.

influence on the removal efficiency of MV by calcium alginate hydrogel beads and magnetic hydrogel beads. Studies on the adsorption equilibrium showed that the ML-F model gives a closer fit to the experimental data than the other used isotherms. This observation is an indicator of heterogeneity of the adsorbent surface. The maximum adsorption capacities of calcium alginate hydrogel beads and magnetic hydrogel beads for the removal of MV were obtained as 889 and $713 \mathrm{mg} / \mathrm{g}$, respectively, which are very high. Also, the findings of kinetic studies showed that the adsorption of MV on calcium alginate hydrogel beads and magnetic hydrogel beads complied with extended pseudo-second-order model. By studying various adsorbent dosages, it was found that the mass of the adsorbent affects the rate of adsorption. Also, the thermodynamic studies revealed that the adsorption of MV on calcium alginate beads is spontaneous, endothermic, and entropy driven. Finally, the advantages of the prepared adsorbents in this study which make them potential adsorbents for practical usage are biocompatibility, ease of separation from the solution, and high adsorption capacity.

\section{EXPERIMENTAL SECTION}

4.1. Materials. $\mathrm{FeCl}_{3} \cdot 6 \mathrm{H}_{2} \mathrm{O}$, urea, ethylene glycol, $\mathrm{CaCl}_{2}$, and MV were purchased from Merck Co.; tetrabutylammonium bromide (TBAB) was a product of Daejung Co; and sodium alginate were purchased from Titra Chem Co. All of the chemicals were used without further purification. In all experiments, deionized water was used.

4.2. Preparation of Calcium Alginate Hydrogel Beads. To prepare calcium alginate hydrogel beads, $1 \mathrm{~g}$ of sodium alginate was dissolved in $50 \mathrm{~mL}$ of deionized water under continuous magnetic stirring to form a transparent and viscous solution. The obtained viscous solution was added drop by drop using a syringe into a $\mathrm{CaCl}_{2}$ aqueous solution $(0.1 \mathrm{~mol}$ $\left.\mathrm{L}^{-1}\right)$. Hydrogel beads were formed instantly and were maintained in $\mathrm{CaCl}_{2}$ solution for $16 \mathrm{~h}$. Then, the calcium hydrogel beads were filtered and washed three times with deionized water to remove any unreacted calcium chloride and kept in deionized water bath. ${ }^{44}$

4.3. Synthesis of Iron Oxide Nanostructures. The preparation of iron oxide was carried out according to a previously reported procedure. ${ }^{33}$ The precursor was prepared by adding $\mathrm{FeCl}_{3} \cdot 6 \mathrm{H}_{2} \mathrm{O}(1.2 \mathrm{~g})$, TBAB $(7.2 \mathrm{~g})$, and urea $(2.7 \mathrm{~g})$ to $180 \mathrm{~mL}$ of ethylene glycol in a $250 \mathrm{~mL}$ round-bottom flask. The obtained red solution was stirred and refluxed at $195{ }^{\circ} \mathrm{C}$ for $45 \mathrm{~min}$ and then cooled to room temperature. The resulting green precipitate was separated by centrifugation and washed four times by ethanol and then calcined at $500{ }^{\circ} \mathrm{C}$ in a furnace under the $\mathrm{N}_{2}$ atmosphere for $3 \mathrm{~h}$ and then cooled to 90 ${ }^{\circ} \mathrm{C}$ in the $\mathrm{N}_{2}$ atmosphere. The obtained powder was cooled to room temperature under ambient condition.
4.4. Preparation of Magnetic Hydrogel Beads. For the preparation of magnetic hydrogel beads, $1 \mathrm{~g}$ of sodium alginate was dissolved in $50 \mathrm{~mL}$ of water; then, $0.5 \mathrm{~g}$ of iron oxide nanoparticles was dispersed in it by sonication, and the mixture was vigorously stirred for $2 \mathrm{~h}$ in a shaker. The viscous suspension was dripped into a $\mathrm{CaCl}_{2}$ bath $\left(0.1 \mathrm{~mol} \mathrm{~L}^{-1}\right)$, and the magnetic hydrogel beads were obtained. To complete the gelation reaction, the magnetic hydrogel beads were allowed to be in contact with the $\mathrm{CaCl}_{2}$ solution for $16 \mathrm{~h}$. The hydrogel magnetic beads were separated from the solution using a magnet, washed three times by deionized water, and then stored in a deionized water bath for further use. ${ }^{44}$

4.5. Characterization. The morphology, composition, and particle size of the prepared iron oxide and dried magnetic composite hydrogel beads were characterized using SEM (TESCAN-VEGA//XMU and TESCAN Vega3), and the elemental identification of the samples was performed by EDX spectroscopy. Also, the crystalline structure of the dried magnetic beads was determined using an X-ray diffraction (XRD) (Bruker AXS-D8 ADVANCE). The presence of various functional groups in samples was identified by FTIR (PerkinElmer, Waltham, MA, USA) using a $\mathrm{KBr}$ pellet, in the range of $4000-400 \mathrm{~cm}^{-1}$.

4.6. Adsorption Experiments. The adsorption of $\mathrm{MV}$ onto calcium alginate hydrogel beads and magnetic hydrogel beads was studied in a batch-mode system. The effect of different parameters including the initial dye concentration, amount of the adsorbent, and temperature on adsorption performance was assessed. The equilibrium experiments were carried out as follows: constant volume of MV solution $(5 \mathrm{~mL})$ with different initial concentrations $(10-500 \mathrm{mg} / \mathrm{L})$ was mixed with a constant amount of wet adsorbent $(0.0972 \mathrm{~g})$. The samples were put in a thermostat shaker $(200 \mathrm{rpm}$ and 25 $\pm 2{ }^{\circ} \mathrm{C}$ ) and shaken for $24 \mathrm{~h}$ to equilibrate. Then, the adsorbent was separated from a solution by a filter or a magnet. The filtrate equilibrium concentration $\left(\mathrm{C}_{\mathrm{e}}\right)$ was measured by using a UV/vis spectrophotometer (PG Instrument LTD model T80) at $585 \mathrm{~nm}$. The adsorbed amount of MV dye per unit mass of the adsorbent was obtained by

$$
q_{\mathrm{e}}=\frac{\left(C_{0}-C_{\mathrm{e}}\right)}{m} \times V
$$

where $C_{0}$ and $C_{\mathrm{e}}$ are the initial and equilibrium concentrations ( $\mathrm{mg} \mathrm{L}^{-1}$ ) of the dye solution, respectively; $m$ is the dried adsorbent mass $(\mathrm{g})$; and $V$ is the solution volume $(\mathrm{L})$.

The kinetics studies were carried out at different initial concentrations of MV $[2,4$, and $8(\mathrm{mg} / \mathrm{L})]$. The experimental setup was the same as that for equilibrium experiments. Here, the residual concentration of $\mathrm{MV}$ was measured at different time intervals, $C_{t}$.

Also, the following equation was used for the determination of the amount of $\mathrm{MV}$ adsorbed $\left[q_{t}(\mathrm{mg} / \mathrm{g})\right]$ at each time interval

$$
q_{t}=\frac{\left(C_{0}-C_{t}\right)}{m} \times V
$$

\section{ASSOCIATED CONTENT}

\section{Supporting Information}

The Supporting Information is available free of charge on the ACS Publications website at DOI: 10.1021/acsomega.8b02498. 
Chemical structure of MV; EDX spectrum of adsorbents; plot of variation of $q$ with time for adsorption of MV onto different dosages of adsorbents; obtained kinetic parameters for the adsorption of MV onto magnetic hydrogel beads; kinetic parameters obtained from PSO and EPSO models for MV adsorption onto both of the adsorbents; table of derived constant parameters of isotherm models for the adsorption of MV onto magnetic hydrogel beads; plot of temperature effect on $\mathrm{MV}$ adsorption by calcium alginate hydrogel beads; $K_{\mathrm{MLF}}$ versus $1 / T$ plot for $\mathrm{MV}$ adsorption by calcium alginate beads; and thermodynamic parameters of MV adsorption by calcium alginate hydrogel beads (PDF)

\section{AUTHOR INFORMATION}

\section{Corresponding Author}

*E-mail: sazizian@basu.ac.ir.

\section{ORCID}

Saeid Azizian: 0000-0003-0040-3478

Notes

The authors declare no competing financial interest.

\section{ACKNOWLEDGMENTS}

The authors are grateful for the financial support from the $\mathrm{Bu}$ Ali Sina University [grant number (32-12-67)].

\section{REFERENCES}

(1) Crini, G. Non-conventional low-cost adsorbents for dye removal: A review. Bioresour. Technol. 2006, 97, 1061-1085.

(2) Pearce, C. I.; Lloyd, J. R.; Guthrie, J. T. The removal of colour from textile Waste water using whole bacterial cells: A review. Dyes Pigm. 2003, 58, 179-196.

(3) Jain, R.; Sikarwar, S. Removal of hazardous dye congored from waste material. J. Hazard. Mater. 2008, 152, 942-948.

(4) Robinson, T.; McMullan, G.; Marchant, R.; Nigam, P. Remediation of dyes in textile effluent: a critical review on current treatment technologies with a proposed alternative. Bioresour. Technol. 2001, 77, 247-255.

(5) Forgacs, E.; Cserháti, T.; Oros, G. Removal of synthetic dyes from wastewaters: a review. Environ. Int. 2004, 30, 953-971.

(6) Mohammadi, A.; Daemi, H.; Barikani, M. Fast removal of malachite green dye using novel superparamagnetic sodium alginatecoated $\mathrm{Fe}_{3} \mathrm{O}_{4}$ nanoparticles. Int. J. Biol. Macromol. 2014, 69, 447-455.

(7) Ali, I.; Asim, M.; Khan, T. A. Low cost adsorbents for the removal of organic pollutants from Waste water. J. Environ. Manage. 2012, 113, 170-183.

(8) Doung, D. D. Adsorption Analysis: Equilibrium and Kinetics; Imperial College Press Singapore: London, 1998; Vol. 2, pp 1-18.

(9) Wu, J.-S.; Liu, C.-H.; Chu, K. H.; Suen, S.-Y. Removal of cationic dye methyl violet $2 \mathrm{~B}$ from water by cation exchange membranes. $J$. Membr. Sci. 2008, 309, 239-245.

(10) Mittal, A.; Gajbe, V.; Mittal, J. Removal and recovery of hazardous triphenylmethane dye, Methyl Violet through adsorption over granulated waste materials. J. Hazard. Mater. 2008, 150, 364375.

(11) Crini, G.; Badot, P.-M. Application of chitosan, a natural aminopolysaccharide, for dye removal from aqueous solutions by adsorption processes using batch studies: a review of recent literature. Prog. Polym. Sci. 2008, 33, 399-447.

(12) Hameed, B. H. Equilibrium and kinetic studies of methyl violet sorption by agricultural waste. J. Hazard. Mater. 2008, 154, 204-212. (13) Doğan, M.; Özdemir, Y.; Alkan, M. Adsorption kinetics andmechanismof cationic methyl violet and methylene blue dyes onto sepiolite. Dyes Pigm. 2007, 75, 701-713.
(14) Mall, I. D.; Srivastava, V. C.; Agarwal, N. K. Removal of Orange-G and Methyl Violet dyes by adsorption onto bagasse fly ashkinetic study and equilibrium isotherm analyses. Dyes Pigm. 2006, 69, 210-223.

(15) Doğan, M.; Alkan, M. Removal of methyl violet fromaqueous solution by perlite. J. Colloid Interface Sci. 2003, 267, 32-41.

(16) Dai, M. Mechanism of adsorption for dyes on activated carbon. J. Colloid Interface Sci. 1998, 198, 6-10.

(17) Hashem, A.; Aly, A. A.; Aly, A. S.; Hebeish, A. Quaternization of cotton stalks and palm tree particles for removal of acid dye from aqueous solutions. Polym.-Plast. Technol. Eng. 2006, 45, 389-394.

(18) Hashem, A. Preparation of a New Adsorbent Based on Wood Pulp for the Removal of Direct Blue 2 from Aqueous Solutions. Polym.-Plast. Technol. Eng. 2006, 45, 779-783.

(19) Mittal, H.; Maity, A.; Ray, S. S. Effective removal of cationic dyes from aqueous solution using gum ghatti-based biodegradable hydrogel. Int. J. Biol. Macromol. 2015, 79, 8-20.

(20) Mittal, H.; Maity, A.; Ray, S. S. Gum karaya based hydrogel nanocomposites for the effective removal of cationic dyes from aqueous solutions. Appl. Surf. Sci. 2016, 364, 917-930.

(21) Saruchi; Kaith, B. S.; Jindal, R.; Kumar, V. Biodegradation of Gum tragacanth acrylic acid based hydrogel and its impact on soil fertility. Polym. Degrad. Stab. 2015, 115, 24-31.

(22) Saruchi; Kaith, B. S.; Jindal, R.; Kumar, V.; Bhatti, M. S. Optimal response surface design of Gum tragacanth-based poly [(acrylic acid)-co-acrylamide] IPN hydrogel for the controlled release of the antihypertensive drug losartan potassium. RSC Adv. 2014, 4, 39822-39829.

(23) Kaith, B. S.; Saruchi; Jindal, R.; Bhatti, M. S. Screening and RSM optimization for synthesis of a Gum tragacanth-acrylic acid based device for in situ controlled cetirizine dihydrochloride release. Soft Matter 2012, 8, 2286-2293.

(24) Mittal, H.; Jindal, R.; Kaith, B. S.; Maity, A.; Ray, S. S. Flocculation and adsorption properties of biodegradable gum-ghattigrafted poly(acrylamide-co-methacrylic acid) hydrogels. Carbohydr. Polym. 2015, 115, 617-628.

(25) Lee, K. Y.; Mooney, D. J. Alginate: properties and biomedical applicatios. Prog. Polym. Sci. 2012, 37, 106-126.

(26) Rocher, V.; Bee, A.; Siaugue, J.-M.; Cabuil, V. Dye removal from aqueous solution by magnetic alginate beads crosslinked with epichlorohydrin. J. Hazard. Mater. 2010, 178, 434-439.

(27) Liu, P.; Jiang, L.; Zhu, L.; Guo, J.; Wang, A. Synthesis of covalently crosslinked attapulgite/poly (acrylic acid-co-acrylamide) nanocomposite hydrogels and their evaluation as adsorbent for heavy metal ions. J. Ind. Eng. Chem. 2015, 23, 188-193.

(28) Sun, X.-F.; Liu, B.; Jing, Z.; Wang, H. Preparation and adsorption property of xylan/poly (acrylic acid) magnetic nanocomposite hydrogel adsorbent. Carbohydr. Polym. 2015, 118, 16-23.

(29) Mittal, H.; Parashar, V.; Mishra, S. B.; Mishra, A. K. $\mathrm{Fe}_{3} \mathrm{O}_{4}$ MNPs and gum xanthan based hydrogels nanocomposites for the efficient capture of malachite green from aqueous solution. Chem. Eng. J. 2014, 255, 471-482.

(30) Zhou, L.; Shao, Y.; Liu, J.; Ye, Z.; Zhang, H.; Ma, J.; Jia, Y.; Gao, W.; Li, Y. Preparation and Characterization of Magnetic Porous Carbon Microspheres for Removal of Methylene Blue by a Heterogeneous Fenton Reaction. ACS Appl. Mater. Interfaces 2014, 6, 7275-7285.

(31) Ma, H.; Li, J.-B.; Liu, W.-W.; Miao, M.; Cheng, B.-J.; Zhu, S.W. Novel synthesis of a versatile magnetic adsorbent derived from corncob for dye removal. Bioresour. Technol. 2015, 190, 13-20.

(32) Lim, S.-F.; Zheng, Y.-M.; Zou, S.-W.; Chen, J. P. Removal of copper by calcium alginate encapsulated magnetic sorbent. Chem. Eng. J. 2009, 152, 509-513.

(33) Zhong, L.-S.; Hu, J.-S.; Liang, H.-P.; Cao, A.-M.; Song, W.-G.; Wan, L.-J. Self-Assembled 3D flowerlike iron oxide nanostructures and their application in water treatment. Adv. Mater. 2006, 18, 24262431. 
(34) Khosravi, M.; Azizian, S. Synthesis of different nanostructured flower-like iron oxides and study of their performance as adsorbent. Adv. Powder Technol. 2014, 25, 1578-1584.

(35) Azizian, S. Kinetic models of sorption: a theoretical analysis. J. Colloid Interface Sci. 2004, 276, 47-52.

(36) Ho, Y. S.; McKay, G. A Comparison of Chemisorption Kinetic Models Applied to Pollutant Removal on Various Sorbents Process Safety. Process Saf. Environ. Prot. 1998, 76, 332-340.

(37) Marczewski, A. W. Application of mixed order rate equations to adsorption of methylene blue on mesoporous carbons. Appl. Surf. Sci. 2010, 256, 5145-5152.

(38) Haerifar, M.; Azizian, S. Fractal-like adsorption kinetics at the solid/solution interface. J. Phys. Chem. C 2012, 116, 13111-13119.

(39) Haerifar, M.; Azizian, S. Fractal-like kinetics for adsorption on heterogeneous solid surfaces. J. Phys. Chem. C 2014, 118, 1129-1134.

(40) Saha, B.; Orvig, C. Biosorbents for hexavalent chromium elimination from industrial and municipal effluents. Coord. Chem. Rev. 2010, 254, 2959-2972.

(41) Eris, S.; Azizian, S. Extension of classical adsorption rate equations using mass of adsorbent: A graphical analysis. Sep. Purif. Technol. 2017, 179, 304-308.

(42) Azizian, S.; Eris, S.; Wilson, L. D. Re-evaluation of the centuryold Langmuir isotherm for modeling adsorption phenomena in solution. Chem. Phys. 2018, 513, 99-104.

(43) Basha, S.; Murthy, Z. V. P.; Jha, B. Sorption of Hg (II) from Aqueous Solutions onto Carica papaya: Application of Isotherms. Ind. Eng. Chem. Res. 2008, 47, 980-986.

(44) Zhu, H.; Fu, Y.; Jiang, R.; Yao, J.; Xiao, L.; Zeng, G. Optimization of copper (II) adsorption onto novel magnetic calcium alginate/maghemite hydrogel beads using response surface methodology. Ind. Eng. Chem. Res. 2014, 53, 4059-4066. 